\title{
10. Über die Begrenzung eines besonderen Gebietes (III).
}

\author{
Von Ken'iti KosekI.
}

(Received Oct. 10, 1948)

Die Struktur der gemeinsamen Begrenzung zweier Gebiete in der Ebene habe ich in der letzten Arbeit" " Über die Begrenzung eines besonderen Gebietes (II) “ behandelt. C. Kuratowski untersuchte auch früher die Struktur dieser Menge. In vorliegenden Arbeit behandeln ich die Beziehung der Kuratowskischen ${ }^{2)}$ Unter: suchungen mit der meinen letzten Mitteilung.

Satz I. Es sei $r$ die beschränkte und gemeinsame Begrenzung zweier Gebiete $\mathfrak{G}_{1}$ und $\mathfrak{S}_{2}$. Alle in einem und demselben Primende ${ }^{3)}$ von $\mathfrak{G}_{1}$ enthaltenen Punkte von $r$ bilden dann ein Häufungskontinuum ${ }^{4}$ in $r$ oder bestehen aus höchstens zwei unzerlegbaren ${ }^{5}$ Kontinuen oder aus einem unzerlegbaren Kontinuum und einem Häufungskontinuum in $t$.

Beweis. Es sei $\left\{\mathscr{H}_{n}\right\}(n=1,2,3, \ldots)$ die Gebietskette, die ein Primende $E$ definiert und durch die Querschnittskette $\left\{q_{n}\right\}(n=1,2,3, \ldots)$ bestimmt wird. Die Menge aller in $E$ enthaltenen Punkte von $r$ soll mit $r_{1}$ bezeichnet werden. Die beiden Endpunkte von $q_{n}$ bezeichnen wir mit $A_{n}$ und $B_{n}$. Hierbei unterscheiden wir die folgenden drei Fälle.

1. Fall. Für unendlich viele $n$ sei weder $A_{n}$ noch $B_{n}$ in $r_{1}$ enthalten. Da eine beliebige Teilfolge von $\left\{q_{n}\right\}$ wiederum das Primende $E$ bestimmt, so können wir annehmen, dass für alle $n$ weder $A_{n}$ noch $B_{n}$ in $r_{1}$ enthalten ist. In diesem Falle muss $r_{1}$ ein Häufungskontinuum in $r$ sein.

Angenommen in der Tat, $r_{1}$ sei kein Häufungskontinuum in $r$. Dann muss $\overline{r-r_{1}} \neq r$ sein, und da $r-r_{1}$ eine zusammenhängende Menge ist, so ist $\overline{r-r_{1}}$ ein

1) K. Koseki: Über die Begrenzung eines besonderen Gebietes (II). Jap. Journ. Math. 19 (1948). Im folgenden zitiere ich diese Arbeit mit (II).

2) C. Kuratowski: Sur la structure des frontières communes à deux régions. Fund. Math. 12 (1928).

3) C. Carathéodory: Über die Begrenzung einfach zusammenhängender Gebiete. Math. Ann. 73 (1913).

4) Ein Teilkontinuum $r_{1}$ von $r$ heisst Häufungskontinuum in $r$, wenn $r-r_{1}$ in $r$ dicht ist.

5) Ein Kontinuum heisst unzerlegbar, wean es nicht Vereinigung zweier echter Teilkontinua ist. 
Kontinuum. Unter der Voraussetzung, dass weder $A_{n}$ noch $B_{n}$ in $r_{1}^{*}$ enthalten ist, muss sowohl $A_{n}$ als auch $B_{n}$ in $\overline{r-r_{1}}$ enthalten sein. Daher gibt es in $\overline{r-r_{1}}$ ein zwischen den beiden Punkten $A_{n}$ und $B_{n}$ irreduzibles Teilkontinuum, das bezeichne ich mit s. Nach dem A. Rosenthalschen Satz ${ }^{1)}$ bestimmt $q_{n}+s$ in der Ebene genau zwei Gebiete $\Re_{1}$ und $\Re_{2}$, die von $q_{n}+s$ begrenzt werden. Man kann leicht einsehen, dass $\mathfrak{S}_{1}-\left(\mathfrak{H}_{n}+q_{n}\right)$ eines von $\mathfrak{T}_{1}$ und $\mathfrak{\Re}_{2}$ identisch ist, und daher ist die Begrenzung von $\mathfrak{S}_{1}-\left(\mathfrak{H}_{n}+q_{n}\right)$ das Kontinuum $q_{n}+s$, da die Begrenzung von $\mathfrak{F}_{2}$ die Punkte von $r_{1}-\left(\overline{r^{2}-r_{1}}\right)$ enthalten muss.

Da $r_{1}-\left(\overline{r-r_{1}}\right)$ eine offene Menge in $r_{1}$ ist, und da die Menge von allen von $\mathfrak{S}_{1}$ aus erreichbaren Punkten überall dicht in $r$ ist, so existieren in $r_{1}-\left(\overline{r-r_{1}}\right)$ unendlich viele Punkte, die von $\mathfrak{S}_{1}$ aus erreichbar sind. Wir wählen die beiden Punkte $C$ und $D$ von dieser Menge aller yon $\mathfrak{S}_{1}$ aus erreichbaren Punkte in $r_{1}-\left(\overline{r-r_{1}}\right)$ aus, so gibt es in $\mathfrak{G}_{1}$ die beiden Einschnitte $e_{1}$ und $e_{2}$ von der Art, dass $e_{1}$ bzw. $e_{2}$ den Punkt $C$ bzw. $D$ als Endpunkt hat. Da weder $C$ noch $D$ in der Begrenzung von $\mathfrak{S}_{1}-\left(\mathfrak{H}_{n}+q_{n}\right)$ enthalten ist, muss sowohl $e_{1}$ als auch $e_{2}$ gegen $E$ konvergieren. ${ }^{3)} \quad$ Dies widerspricht aber dem C. Carathéodoryschen Satz. Folglich. muss im 1. Falle $r_{1}$ ein Häufungskontinuum in $r$ sein.

2. Fall. Für unendlich viele $n$ sei sowohl $A_{n}$ als auch $B_{n}$ in $r_{1}$ enthalten. Da eine beliebige Teilfolge von $\left\{q_{n}\right\}$ wiederum das Primende $E$ bestimmt, so können wir annehmen, dass für alle $n$ sowohl $A_{n}$ als $B_{n}$ in $r_{1}$ enthalten ist. In diesem Falle muss $r_{1}$ entweder ein Häufungskontinuum in $r$ oder die Summe von höchstens zwei unzerlegbaren Kontinuen sein.

Angenommen, $r_{1}$ sei kein Häufungskontinuum in $r$. Wenn $r_{1}$ nicht ein unzerlegbares Kontinuum ist, so wird $r_{1}$ in zwei echte Teilkontinua $s$ und $t$ zerlegt. Jedes Primende von $\mathfrak{S}_{1}$ kann durch eine Kette von Querschnitten bestimmt werden, die auf konzentrischen Kreisen liegen, und gegen den gemeinsamen Mittelpunkt dieser Kreise konvergieren. Daher kann man anfangs $q_{n}$ konstruieren derart, dass $q_{n}(n=1,2,3, \ldots)$ ein kreisförmiger Querschnitt mit einem Punkt $A$ von $\tau^{*}$ als Mittelpunkt ist. Dann muss der Punkt $A$ in $s \cdot t$ enthalten sein.

Angenommen in der Tat, dass der Punkt $A$ in einem und nur einem von den beiden $s$ und $t$, etwa in $s$, enthalten ist. Da $q_{n}$ den Punkt $A_{n}$ mit dem Punkte $B_{n}$ (1919).

1) A. Rosenthal: Teilung der Ebene durch irreduzible Kontinua. Sgb. Bay. Ak. Wiss.

2) $\mathrm{Vgl}$ (II).

3) $\mathrm{Vgl}(\mathrm{II})$. 
verbindet, und da der Durchmesser von $q_{n}$ mit $n \rightarrow \infty$ gegen 0 konvergiert, so muss für ein genügend grosses $n$ sowohl $A_{n}$ als auch $B_{n}$ in $s$ enthalten sein. Wenn wir ein zwischen $A_{n}$ und $B_{n}$ irreduzibles Teilkontinuum von $s$ mit $l$ bezeichnen, so bestimmt nach dem A. Rosenthalschen Satze $l+q_{n}$ in der Ebene genau zwei Gebiete, die von dem ganzen Kontinuum $l+q_{n}$ begrenzt werden. Eines von diesen beiden Gebieten muss mit $\mathfrak{S}_{1}-\left(\mathfrak{H}_{n}+q_{n}\right)$ übereinstimmen, da die Begrenzung von $\mathfrak{H}_{n}$ die Punkte von $t$ enthalten muss. Daraus folgt, dass sowohl $C$ als auch $D$ ein erreichbarer Punkt des Primendes $E$ sein muss, wenn wir zwei Punkte $C$ und $D$ aus $r-s$ wählen, die von $\mathfrak{S}_{1}$ aus erreichbar sind und die nicht Grenzpunkte von $s$ sind. Das widerspricht aber dem C. Carathéodoryschen Satz, damit muss der Punkt $A$ in $s \cdot t$ enthalten sein. Ebenfalls kann es sich niemals ergeben, dass für unendlich viele $n$ eines von beiden $s$ und $t$ die beiden Punkte $A_{n}$ und $B_{n}$ gleichzeitig enthält.

Zweitens bezeichnen wir $r_{1}-t$ mit $s_{1}$ und beweisen, dass die abgeschlossene Hülle $\bar{s}_{1}$ von $s_{1}$ ein unzerlegbares Kontinuum ist. Die Menge $s_{1}$ muss zunächst eine zusammenhängende Menge sein. Angenommen in der Tat, dass $s_{1}$ keine zusammenhängende Menge ist, dann muss $s_{1}$ in die beiden Teilmengen $s_{2}$ und $s_{3}$ zerlegt wer. den derart, dass $s_{2}$ und $s_{3}$ punktfremd sind und keine einen Häufungspunkt der anderen enthält. Alsdann ist sowohl $t+\varepsilon_{2}$ als $t+\varepsilon_{3}$ ein Kontinuum. Da $t$ für fast alle $n$ einen und nur einen von den beiden $A_{n}$ und $B_{n}$ enthalten muss, so muss eines von den beiden $t+s_{2}$ und $t+s_{3}$, etwa $t+s_{3}$, für unendlich viele $n$ die beiden Punkte $A_{n}$ und $B_{n}$ enthalten. Wir bezeichnen ein zwischen $A_{n}$ und $B_{n}$ irreduzibles Teilkontinum von $t+s_{3}$ mit $l$, so bestimmt $l+q_{n}$ in der Ebene genau zwei Gebiete $\Re_{1}$ und $\AA_{2}$, die von dem ganzen Kontinuum $l+q_{n}$ begrenzt werden. Man kann leicht dann einsehen, dass $\mathbb{G}_{1}-\left(\mathfrak{H}_{n}+q_{n}\right)$ mit einem von beiden $\Re_{1}$ und $\Re_{2}$ identisch ist, da die Begrenzung von $\mathfrak{H}_{n}$ alle Punkte von $\varsigma_{2}$ enthält. Daraus folgt, dass, wenn wir die beiden Punkte $C$ und $D$ von $r-\left(t+s_{3}\right)$ auswählen, die von $\mathfrak{G}_{1}$ aus erreichbar sind, sowohl $C$ als auch $D$ ein erreichbarer Punkt von $E$ sein muss. Das widerspricht aber dem Carathéodoryschen Satz. Damit ist bewiesen, dass $s_{\mathbf{1}}$ eine zusammenhängende Menge ist.

Wir beweisen nun, dass $\vec{s}_{1}$ ein unzerlegbares Kontinuum ist. Angenommen in (ler Tat, dass $\bar{s}_{1}$ kein unzerlegbares Kontinuum ist, so muss $\bar{s}_{1}$ in die beiden echten Teilkontinua $u_{1}$ und $u_{2}$ zerlegt werden. Dabei muss weder $u_{1}-u_{2}$ noch $u_{2}-u_{1}$ in $t$ enthalten sein. Angenommen in der Tat, dass eines von den beiden $u_{1}-u_{2}$ und $u_{2}-u_{1}$, etwa $u_{1}-u_{2}$, in $t$ enthalten ist. Dann muss $u_{1}-u_{2}$ in $\bar{s}_{1}-s_{1}$ enthalten sein, 
und daher muss $s_{1}$ in $u_{2}$ enthalten sein. Daraus folgt; dass alle Punkte von $u_{1}-u_{2}$ : die Häufungspunkte von $u_{2}$ sind. Das widerspricht aber der Eigenschaft von $u_{2}$. Wir bekommen also die beiden Kontinua $t+u_{1}$ und $t+u_{2}$ und zwar von der Art, dass kein von den beiden Kontinuen $t+\iota_{1}$ und $t+u_{2}$ in andern enthalten ist. Da $r_{1}=t+\bar{s}_{1}$ ist und sowohl $t$ als auch $\bar{s}_{1}$ ein echtes Teilkontinuum von $r_{1}$ ist, kann es sich nicht ergeben, dass eines von $t$ und $\bar{s}_{1}$ für unendlich viele $n$ die beiden Punkte $A_{n}$ und $B_{n}$ gleichzeitig enthält. Folglich muss mindestens eines von $t+u_{1}$ und $t+u_{2}$ für unendlich viele $n$ die beiden Punkte $A_{n}$ und $B_{n}$ enthalten. Anderseits, da $r_{1}$ in die zwei echte Teilkontinua $t+u_{1}$ und $t+u_{2}$ zerlegt wird, so kann es sich niemals ergeben, dass eines von $t+u_{x}$ und $t+u_{2}$ für unendlich viele $n$ die beiden Punkte $A_{n}$ und $B_{n}$ enthält. Also gelangen wir unter der Annahme, dass $\bar{s}_{1}$ nicht ein unzerlegbares Kontinuum ist, zu einem Widerspruch. Daher muss $\bar{s}_{1}$ ein unzerlegbares Kontinuum sein.

Wenn wir nun $r_{1}-\bar{s}_{1}$ mit $t_{1}$ bezeichnen, so können wir ebenfalls beweisen, dass $\bar{t}_{1}$ ein unzerlegbares Kontinuum ist. Also muss $\nu_{1}$ aus zwei unzerlegbaren Kontinuen $\bar{t}_{1}$ und $\bar{s}_{1}$ béstehen, wie behauptet, wenn $r_{1}$ weder ein Häufungskontinuum in $r$ noch ein unzerlegbares Kontinuum ist.

3. Fall. Für fast alle $n$ sei einer von den beiden $A_{n}$ und $B_{n}$ und nicht der andere in $r_{1}$ enthalten. In diesem Falle muss $r_{1}$ entweder ein Häufungskontinuum in $r$ oder ein unzerlegbares Kontinuum oder die Vereinigungsmenge eines unzerlegbaren Kontinuum und eines Häufungskontinuum in $r$ sein. Wie im 2 . Falle konstruieren wir $q_{n}$, derart dass $q_{n}(n=1,2, \ldots)$ ein kreisformiger Querschnitt mit einem Punkt $A$ von $\imath^{*}$ als Mittelpunkt ist.

Angenommen, dass $r_{1}$ kein Häufungskontinuum in $r$ ist. Um es hierbei zu beweisen, dass $r_{1}$ entweder ein unzerlegbares Kontinuum oder die Vereinigungsmenge eines unzerlegbaren Kontinuum und eines Häufungskontinuum in $r$ ist, zeigen wir zunächst, dass $\overline{r-\left(r-r_{x}\right)}$ ein unzerlegbares Kontinuum ist.

In ganz analoger Weise wie im 2. Falle können wir zeigen, dass der Punkt $A$ in $\overline{\left(r-r_{1}\right)} \cdot \overline{r-\left(r-r_{1}\right)}$ enthalten ist. Wenn das Kontinuum $\overline{r-\left(\overline{r-r_{1}}\right)}$ kein unzerlegbares Kontinuum ist, so wird $\overline{r-\left(\overline{r-r_{1}}\right)}$ in zwei echte Teilkontinua $s$ und $t$ zerlegt. Weder $s$ noch $t$ muss dann in $\overline{r-r_{1}}$ enthalten sein; denn, wenn etwa $s$ in $\overline{r-r_{1}}$ enthalten ist, so müssen alle Punkte von $s$ die Häufungspunkte von $t$ sein, da alle Punkte von $\left(\overline{\left.r-r_{1}\right)} \cdot \overline{r-\left(\overline{r-r_{1}}\right)}\right.$ die Häufungspunkte von $r-\overline{\left(r-r_{1}\right)}$ sind. 
Mindestens eines von den beiden $\overline{r-r_{1}}+s$ und $\overline{r-r_{1}}+t$, etwa $\overline{r-r_{1}}+s$, muss für unendlich viele $n A_{n}$ und $B_{n}$ enthalten, da nach der Voraussetzung im 3 . Falle $s+t$ nicht gleichzeitig $A_{n}$ und $B_{n}$ enthält. Wir bezeichnen nun ein zwischen $A_{n}$ und $B_{n}$ irreduzibles Teilkontinuum von $\overline{r-r_{1}}+s$ mit $l$, so bestimmt $l+q_{n}$ in der Ebene genau zwei Gebiete $\Re_{\mathbf{1}}$ und $\Re_{2}$, die von $l+q_{n}$ begrenzt werden. Eines von den beiden $\Re_{1}$ und $\Re_{2}$ muss mit $\mathfrak{S}_{1}-\left(\mathscr{L}_{n}+q_{n}\right)$ übereinstimmen, da die Begrenzung von $\mathfrak{S}_{n}$ alle Punkte $t$ enthalten muss. Daraus folgt, dass, wenn wir zwei Punkte $C$ und $D$ von $t$ auswählen, die von $\mathcal{S}_{1}$ aus erreichbar sind und nicht in $\overline{r-r_{1}}+s$ enthalten sind, sowohl $C$ als auch $D$ ein erreichbarer Punkt vom Primende $E$ sein muss. Das widerspricht aber dem Carathéodoryschen Satz. Also kommen wir unter der Annahme, dass $\overline{r-\left(\overline{\left.r-r_{1}\right)}\right)}$ kein unzerlegbares Kontinuum ist, zu einem Widerspruch, damit ist bewiesen, dass $\overline{r-\left(\overline{r-r_{1}}\right)}$ ein unzerlegbares Kontinuum ist.

Wenn $\overline{r-\left(\overline{r-r_{1}}\right)}$ mit $r_{1}$ identisch ist, so ist $r_{1}$ ein unzerlegbares Kontinuum. Angenommen nun, dass $\overline{r-\left(\overline{r-r_{1}}\right)}$ mit $r_{1}$ nicht identisch ist. Wir setzen einen Punkt $B$ von $r-r_{1}$ fest, so enthält für ein genügend grosses $n$ die Begrenzung von $\mathfrak{H}_{n}$ nicht den Punkt $B$. Den Durchschnitt der Begrenzung von $\mathscr{H}_{n}$ mit $v$ bezeichnen wir mit $p_{n}$, so ist $p_{n}$ ein Kontinuum. Das Kontinuum $p_{n}$ enthält $r_{1}$ und damit auch $\overline{r-\left(\overline{r-r_{1}}\right)}$. Nach der Annahme, dass $\overline{r-\left(r-r_{1}\right)}$ mit $r_{1}$ nicht identisch ist, ist $p_{n}-\overline{r-\left(\overline{r-r_{1}}\right)}$ nicht die Nullmenge.

Nach der Voraussetzung im 3. Falle kann es sich niemals ergeben, dass sowohl $A_{n}$ als auch $B_{n}$ in $\overline{r-\left(\overline{r-r_{i}}\right)}$ enthalten ist. Das kann auch sich nicht ergeben, dass $A_{n}$ und $B_{n}$ gleichzeitig in $r-\overline{r-\left(\overline{r-r_{1}}\right)}$ enthalten sind. Angenommen in der Tat, dass $r-\overline{r-\left(\overline{r-r_{1}}\right)}$ sowohl $A_{n}$ als $B_{n}$ enthält, so muss $\overline{r-r_{1}}$ aurh die beiden Punkte $A_{n}$ und $B_{n}$ enthalten. Wir bezeichnen ein zwischen $A_{n}$ und $B_{n}$ irreduzibles Teilkontinuum von $\overline{r-r_{1}}$ mit $l$, so bestimmt $l+q_{n}$ in der Ebene genau zwei Gebiete $\Re_{1}$ und $\Re_{2}$, die von $l+q_{n}$ begrenzt werden. Eines von den beiden Gebieten $\Re_{\mathbf{1}}$ und $\Re_{\mathbf{2}}$ muss mit $\mathfrak{S}_{1}-\left(\mathfrak{H}_{n}+q_{n}\right)$ identisch sein, da die Begrenzung von $\mathfrak{H}_{n}$ alle Punkte von $r_{1}$ enthalten muss. Daraus folgt, dass, wenn wir zwei Punkte $C$ und $D$ von $r_{1}-\left(\overline{r-r_{1}}\right)$ auswählen, die von $\mathfrak{G}_{1}$ aus erreichbar sind, sowohl $C$ als auch $D$ ein erreichbarer Punkt vom Primende $E$ sein muss. Das widerspricht aber dem Carathéodoryschen Satz. Daher muss einer von $A_{n}$ und $B_{n}$ in $\overline{r-\left(r-r_{1}\right)}$ und der andere in $r-\overline{r-\left(\overline{r-r_{1}}\right)}$, enthalten sein.

Wir bezeichnen nun ein zwischen $A_{n}$ und $B_{n}$ irreduzibles Teilkontinuum von $p_{n}$ mit $l_{n}$, so bestimmt $l_{n}+q_{n}$ in der Ebene genau zwei Gebiete $\Re_{1}$ und $\Re_{2}$, die von 
dem ganzen Kontinuum $l_{n}+q_{n}$ begrenzt werden. Eines von den beiden Gebieten $\Re_{1}$ und $\Re_{2}$ muss mit $\mathfrak{H}_{n}$ identisch sein, da die Begrenzung von $\mathfrak{S}_{1}-\left(\mathfrak{H}_{n}+q_{n}\right)$ den Punkt $B$ enthalten muss. Daraus folgt, dass $p_{n}$ mit $l_{n}$ identisch ist und daher ein zwischen $A_{n}$ und $B_{n}$ irreduzibles Kontinuum ist.

Da $\left.\overline{r-\left(r-r_{1}\right.}\right)$ einen von den beiden $A_{n}$ und $B_{n}$ und nicht den andern enthält, so muss $\left.p_{n}-\overline{r-\left(r-r_{1}\right.}\right)$ eine zusammenhängende Menge sain. Angenommen in der Tat, dass $p_{n}-\overline{r-\left(r-r_{1}\right)}$ nicht eine zusammenhängende Menge ist, so wird $p_{n}-\overline{r-\left(r-r_{1}\right)}$ in zwei Teilmengen $u$ und $v$ zerlegt derart, dass $u$ und $v$ zueinander punktfremd sind und keine einen Häufungspunkt der andern enthält. Alsdann muss eines von den beiden Kontinuen $\overline{r-\left(r-r_{1}\right)}+u$ und $\overline{r-\left(r-r_{1}\right)}+v$ die beiden Punkte $A_{n}$ und $B_{n}$ enthalten; das widerspricht aber der Tatsache, dass $p_{n}$ ein zwischen $A_{n}$ und $B_{n}$ irreduzibles Kontinuum ist.

Wir bezeichnen $p_{n}-\overline{r^{*}-\left(\overline{r-r_{1}}\right)}$ mit $k_{n}$, dann ist die abgeschlossene Hülle $\bar{k}_{n}$ ein Kontinuum. Wir konstruieren für fast alle $n(n \geqq m)$ das Kontinuum $\overline{k_{n}}$. Es entsteht, dass $\left.r_{1}=\prod_{n=m}^{\infty} p_{n}=\prod_{n=m}^{\infty} \overline{\left(r-r-r_{1}\right.}+\overline{k_{n}}\right)=\overline{r-\left(r-r_{1}\right)}+\prod_{n=m}^{\infty} \overline{k_{n}}$ ist. Das Kontinuum $\vec{k}_{n}$ ist in $p_{n}-\overline{r-\left(\overline{\left.r-r_{1}\right)}\right\}}$ und daher in $\overline{r-r_{1}}$ enthalten. Anderseits ist $\prod_{n=m}^{\infty} \bar{k}_{n}$ in $r_{\mathbf{1}}$ enthalten, so $\prod_{n=m}^{\infty} \bar{k}_{n}$ ist ein Häufungskontinuum in $\overline{r-r_{1}}$ und damit auch in $r$. So ist $r_{1}$ die Vereinigungsmenge von dem unzerlegbaren Kontinuum $\overline{r-\left(r-r^{2}\right)_{1}}$ und dem Häufungskontinuum $\prod_{n=m}^{\infty} k_{n}$ in $r^{2}$.

Satz. II. Es sei $r$ die beschränkte und gemeinsame Begrenzung zweier Gebiete $\mathfrak{B}_{1}$ und $\mathfrak{S}_{2}$, Jenachdem $r$ die Vereinigungsmenge von höchstens abzählbarer Anzahl von Häufungskontinuen in $r$ und unzerlegbaren Kontinuen ist oder nicht, ist $r^{*}$, monostratique " oder nicht.

Beweis. Wie C. Carathéodory bewiesen hat, gibt es eine umkehrbar eindeutige und stetige Abbildung der Primenden des Gebietes $\mathcal{G}_{1}$ auf alle Punkte einer Kreisperipherie $k$. Wir bezeichnen im folgenden einen Punkt von $k$ mit $x, x^{\prime}, \ldots$ oder $y, y^{\prime}, \ldots$ und das zu $x, x^{\prime}, \ldots$ entsprechende Primende mit $E_{x}, E_{x^{\prime}}, \ldots$. Erstens unterscheiden wir zwei Fälle.

Fall A. $\quad r$ sei nicht die Vereinigungsmenge von höchstens abzählbarer Anzahl von Häufungskontinuen in $r$ und unzerlegbaren Kontinuen.

1. Schritt. Es seien $x$ und $x_{1}$ zwei Punkte von $k$ derart, dass $E_{x}$ und $E_{x_{1}}$ die Punkte von $r$ gemeinsam enthalten. Dann wird $k$ durch die beiden Punkte $x$ und 
$x_{1}$ in die beiden abgeschlossenen Kreisbogen $\underset{x}{2}$ und $x_{1} x$ derart zerlegen, dass $2 x_{1}$ und $\underset{x_{1} x}{\curvearrowleft}$, bis auf Endpunkte, keine Punkte gemein haben. Nach dem Satze IV in meiner letzten Arbeit (II) besitzt einer von den beiden $\widetilde{x} x_{1}$ und $\widetilde{x_{1} x}$, etwa $\tilde{x} x_{\mathbf{1}}$, die folgende Eigenschafti); ein beliebiger Punkt von $2 x_{1}$ entspricht dem mehrfachen Primende; genauer gesagt, wenn $y$ einen beliebigen Punkt auf $\widetilde{\curvearrowleft} x_{1}$ bedeutet, und wenn $A$ ein in $E_{y}$ enthaltener Punkt von $\tau^{*}$ ist, so muss $A$ in mindestens einem von den beiden $E_{x}$ und $E_{x_{1}}$ enthalten sein. Das kann aber sich nicht ergeben, dass sowohl $\varkappa_{x}$ wie auch $\check{x}_{\mathbf{1}} x$ die obige Eigenschaft IV von (II) hat. Wir bezeichnen ${ }^{2}$ in der Tat mit $m_{x}$ die Menge aller in $E_{x}$ enthaltenen Punkte von $r$ und mit $m_{x_{1}}$ die Nenge aller in $E_{x_{1}}$ enthaltenen Punkte von $r$, so muss $r$ mit der Vereinigung von $m_{x}$ und $m_{x_{1}}$ übereinstimmen, damit muss $\eta^{*}$ nach Satz I die Vereinigungsmenge von höchstens abzählbarer Anzahl von unzerlegbaren Kontinuen und Häufungskontinuen in $\tau^{r}$ sein. Das kann aber im Falle A nicht auftreten. Wir sagen im folgenden: der Punkt $x_{1}$ in oben oder in unten für $x$ liegt, jenachdem $x_{1} x$ oder $x_{x}$ die Eigenschaft IV von (II) hat.

Ein Punkt $A$ von $r$ sei in einem Primende $E_{x}$ enthalten. Wenn der Punkt $A$ nicht einfacher ${ }^{3)}$ Punkt von $E_{x}$ ist, so bestimmt die Menge aller Punkte $x^{\prime}$ von $k$ von der Art, dass das zu $x^{\prime}$ entsprechende Primende $E_{x^{\prime}}$ den Punkt $A$ enthält und $x^{\prime}$ in oben für $x$ liegt, eine obere Grenze $x_{1}$. Das Primende $E_{x_{1}}$ enthält dann den Punkt A. Es gibt in der Tat eine Punktfolge $\left\{y_{n}\right\}(n=1,2,3, \ldots)$ auf $k$ von der Art, dass das zu $y_{n}$ entsprechende Primende $E_{y_{n}}$ den Punkt $A$ enthält und $y_{n}$ in oben für $x$ liegt, und dass $\left\{y_{n}\right\}$ mit $n \rightarrow \infty$ gegen den Punkt $x_{1}$ konvergiert. Da es eine umkehrbar eindeutige und stetige Abbildung der Primenden von $\mathfrak{G}_{1}$ auf alle Punkte von $k$ gibt, so konvergiert die Primendenfolge $\left\{E_{y_{32}}\right\}$ mit $n \rightarrow \infty$ gegen $E_{x_{1}}$. $\left\{\mathfrak{S}_{x_{1}}^{m}\right\}(m=1,2,3, \ldots)$ sei die das Primende $E_{x_{1}}$ definierende Gebietskette, so gehören $^{4)}$ fast alle Primenden der Folge $\left\{E_{y_{22}}\right\}$ zu jedem. Gebiet $\mathfrak{F}_{x_{1}}^{m}$. Da jedes Primende $E_{y_{a x}}$ den Punkt $A$ enthält, so enthält die abgeschlossene Hülle $\overline{\mathfrak{Y}}_{x_{1}}^{m}(m=1$, $\cdot 2, \ldots$ ) auch den Punkt $A$.

1) Diese Eigenschaft bezeichnen wir im folgenden als die Eigenschaft IV von (II).

2) Im folgenden bezeichnen wir stets die Menge aller in einem Primende $E_{i c}$ enthaltenen Punkte mit $m_{c}$.

3) Ein Punkt $A$ der Begrenzung $r$ von $\mathfrak{S}_{1}$ wird einiach genannt, wenn er in nur einem ein:igen Primende von $\mathfrak{G}_{1}$ enthalten ist. Je ein Punkt von $r$, der nicht einfach ist, heisst mehrfacher Punkt.

4) Es sei (s) einfach zusammenhängendes Gebiet und (G' ein Teilgebiet von (Bs. $E_{x}$ sei ein Primende von of und $\left\{\mathfrak{S}_{2}\right\}(n=1,2, \ldots)$ die das Primende $E_{x}$ definierende Gebietskette. Wenn fast alle Gebiete von $\left\{\mathfrak{S}_{2 n}\right\}$ in $\mathcal{G}^{\prime}$ enthalten sind, so sagen wir, dass Primende $E_{c}$ zu (3)' gehört. 
Zweitens liegt der Punkt $x_{1}$ in oben für $x$. Angenommen in der Tat, dass $x$ in oben für $x_{1}$ liegt, so muss $\gamma^{*}$ die Vereinigungsmenge $m_{x}+m_{x_{1}}+m_{y_{1}}+m_{y_{2}}+\cdots$ sein. Daraus folgt nach Satz I, dass $\eta^{*}$ die Vereinigungsmenge von höchstens abzählbarer Anzahl von unzerlegbaren Kontinuen und Häufungskontinuen in $v^{*}$ ist. Das widerspricht aber der Voraussetzung im Falle A.

Ebenfalls bestimmt die Menge aller Punkte $x^{\prime \prime}$ von $k$, so dass das zu $x^{\prime \prime}$ entsprechende Primende $E_{x^{\prime \prime}}$ den Punkt $A$ enthält und $x$ in oben für $x^{\prime \prime}$ liegt, eine untere Grenze $x_{2}$. Das Primende $E_{x_{2}}$ enthält dann den Punkt A und $x$ liegt in oben für $x_{2}$. Wenn $x_{2}$ nicht mit $x$ übereinstimmt, so kann $x_{2}$ niemals auf $x_{1} x$ liegen. Angenommen in der Tat, dass $x_{2}$ auf $x_{1} x$ liegt, so muss $r$ die Vereinigungsmenge $m_{x}+m_{x_{1}}+m_{x_{2}}$ sein. Das kann im Falle A nicht der Fall sein.

Wir bezeichnen mit $\widetilde{x}_{0}$ die untere Grenze der Menge aller Punkte $x^{\prime}$ von $k$ von der Art, dass $E_{x_{1}}$ und $E_{x^{\prime}}$ gemeinsame Punkte enthalten und $x_{1}$ in oben für $x^{\prime}$ liegt, und mit $\widetilde{x}_{1}$ die obere Grenze der Menge aller Punkte $x^{\prime \prime}$ von $k$ von der Art, dass $E_{\tilde{x}_{0}}$ und $E_{x^{\prime \prime}}$ gemeinsame Punkte enthalten und $x^{\prime \prime}$ in oben für $\widetilde{x}_{0}$ liegt. In ganz analoger Weise wie oben können wị leicht zeigen, dass $E_{\tilde{x}_{0}}$ und $E_{\tilde{x}_{1}}$ die gemeinsame Punkte enthalten und $\widetilde{x}_{1}$ in oben für $\widetilde{x}_{0}$ liegt.

Hierbei können wir die folgenden vier Möglichkeiten voraussehen.

1. Fall. $x_{1}=\widetilde{x}_{1}$ und $x_{2}=\widetilde{x}_{0}$

2. Fall. $x_{1}=\widetilde{x}_{1}$ und $x_{2} \widetilde{x}_{0}$

3. Fall. $x_{1} \neq \widetilde{x}_{1}$ und $x_{2}=\widetilde{x}_{0}$

4. Fall. $x_{1} \neq \widetilde{x}_{1}$ und $x_{2} \neq \widetilde{x}_{0}$.

In Wirklichkeit kann 4. Fall nicht auftreten. Denn mindestens eines von den beiden $E_{\tilde{x}_{1}}$ und $E_{\tilde{x}_{0}}$ muss den Punkt $A$ enthalten. ${ }^{1)}$

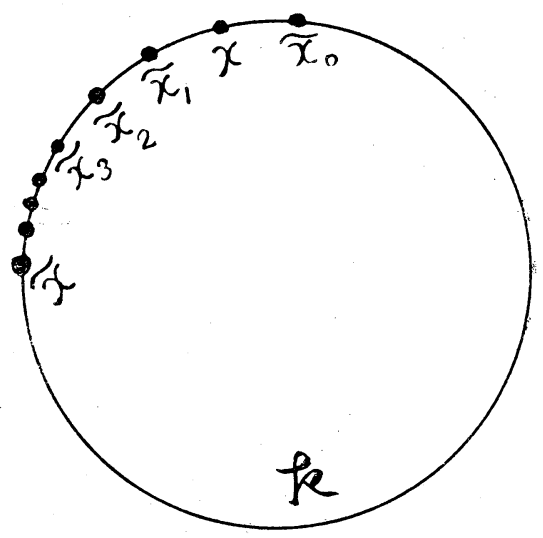

Nächstens bezeichnen wir mit $\widetilde{x}_{2}$ die obere Grenze der Menge aller Punkte $x^{\prime}$ von $k$ von der Art, dass $E_{\tilde{x}_{1}}$ und $E_{x^{\prime}}$ gemeinsame Punkte enthalten und $x^{\prime}$ in oben für $\widetilde{x}_{1}$ liegt, und mit $\widetilde{x}_{3}$ die obere Grenze der Menge aller Punkte $x^{\prime}$ von $k$ von der Art, dass $E_{\tilde{x}_{2}}$ und $E_{x^{\prime}}$ gemeinsame Punkte enthalten und $x^{\prime}$ in oben für $\widetilde{x}_{2}$ liegt.

1) Die untere Grenze $\check{x}_{0}{ }^{*}$ von der Menge aller Punkte $x^{\prime \prime}$ von $k$ von der Art, dass das zu $x^{\prime \prime}$ entsprechende Primende $E_{x^{\prime \prime}}$ und das Primende $E_{x_{1}}$ gemeinsame Punkte enthalten und $\check{x}_{1}$ in oben für $x^{\prime \prime}$ liegt, muss mit $\tilde{x}_{0}$ übereinstimmen. Dies ist ebenfalls leicht einzusehen. 
Dabei ist es zu bemerken, dass $\underset{\widetilde{x}_{2} \widetilde{x}_{1}}{\mathscr{\leftarrow}}$ und $\underset{\widetilde{x}_{3} \widetilde{x}_{2}}{\mathscr{}}$ nur einen einzigen Punkt $\widetilde{x}_{2}$ gemeinsam hat.

Diese Verfahren setzen wir fort, dann bekommen wir eine Folge der Punkte$\widetilde{x}_{1}, \widetilde{x}_{2}, \widetilde{x}_{3}, \ldots$. Wir bezeichnen $\lim _{n \rightarrow \infty} \widetilde{x}_{n}$ mit $\widetilde{x}$.

In gleicher Weise bezeichnen wir mit $\widetilde{x}_{-1}$ die untere Grenze der Menge aller Punkte $x^{\prime}$ von $k$ von der Art, dass $E_{\tilde{x}_{0}}$ und $E_{x^{\prime}}$ gemeinsame Punkte enthalten und $\widetilde{x}_{0}$ in oben für $x^{\prime}$ liegt. Diese Verfahren setzen wir fort, dann bekommen wir eine Folge der Punkte $\widetilde{x}_{-1}, \widetilde{x}_{-2}, \widetilde{x}_{-3}, \ldots$ Wir bezeichnen $\lim _{\imath \rightarrow \infty} \widetilde{x}_{-n}$ mit $\widetilde{x}$. Der Punkt $\widetilde{x}$ kann nicht $\widetilde{x}$ übereinstimmen. Denn, wenn $\widetilde{x}$ mit $\ddot{\widetilde{x}}$ übereinstimmt, so muss $r^{\circ}$ die Vereinigungsmenge $m_{\tilde{x}_{0}}+m_{\tilde{x}_{1}}+m_{\tilde{x}_{2}}+\cdots+m_{\tilde{x}_{-1}}+m_{\tilde{x}_{-2}}+\cdots$ sein; das widerspricht aber der Voraussetzung des Falles A. Damit bekommen wir ein abgeschlossenes Intervall $\widetilde{\widetilde{x}} \widetilde{\widetilde{x}}$ auf $k$.

2. Schritt. Für einen Punkt $x$ von $k$ definieren wir wie im 1. Schritte $\left\{\widetilde{x}_{n}\right\}(n=0, \pm 1, \pm 2, \pm 3, \cdots), \widetilde{x}$ und $\widetilde{x}$. Es seien $y_{1}$ und $y_{2}$ zwei Punkte von $k$ von der Art, dass $\boldsymbol{E}_{v_{1}}$ und $\boldsymbol{E}_{y_{2}}$ gemeinsame Punkte enthalten und $y_{1}$ in oben für $y_{2}$ liegt. Wenn $y_{2}$ auf $\widetilde{x}_{p} \widetilde{x}_{p-1}$ und $\widetilde{x}_{p}$ auf $\widetilde{y}_{1} y_{2}$ liegt, und wenn $\widetilde{x}_{p}$ nicht mit $y_{2}$ übereinstimmt, so muss $y_{1}$ auf $\widetilde{x}_{p+1} \widetilde{x}_{p}$ liegen. Um dies zu beweisen, unterscheiden wir zwei Fälle.

\section{Fall a) $p \geqq 1$.}

Angenommen in diesem Falle, dass $\widetilde{x}_{p+1}$ auf $\widetilde{y}_{1} y_{2}$ liegt und $\widetilde{x}_{p+1}$ nicht mit $y_{\mathbf{r}}$ übereinstimmt. Dann können nicht alle in $\boldsymbol{E}_{y_{2}}$ enthaltenen Punkte in $\boldsymbol{E}_{\tilde{x}_{p}}$ enthalten sein. Daher müssen alle in $E_{y_{2}}$ und nicht in $E_{\tilde{x}_{p}}$ enthaltenen Punkte in $E_{\tilde{x}_{p-1}}$ enthalten sein. Hieraus folgt, dass alle sowohl in $E_{y_{1}}$ als in $E_{y_{2}}$ enthaltenen Punktein $E_{x_{p-1}}$ enthalten sind, das ist aber ein Widerspruch mit der Eigenschaft von $\widetilde{x}_{p}$. Damit ist die Behauptung bewiesen.

Fall b) $p \leqq 0$.

Der Punkt $\widetilde{x}_{1}$ kann nicht auf $\widetilde{y}_{1} y_{2}$ liegen. Wenn $\widetilde{x}_{1}$ auf $\mathscr{y}_{1} y_{2}$ liegt, so muss $\widetilde{x}_{0}$. auch auf $\mathscr{y}_{1} y_{2}$ liegen. Ein Punkt $A$ sei sowohl in $E_{\tilde{x}_{1}}$ als auch in $E_{\tilde{x}_{0}}$ enthalten, so muss der Punkt $A$ in $E_{y_{1}}$ oder in $E_{y_{2}}$ enthalten sein. Da $y_{2}$ auf $\widetilde{x}_{p} \widetilde{x}_{p-1}$ liegt und $\widetilde{x}_{p}$ nicht mit $y_{2}$ übereinstimmt, so muss der Punkt $A$ in $E_{y_{1}}$ enthalten sein. Folglich muss $y_{1}$ mit $\widetilde{x}_{1}$ übereinstimmen; damit muss $y_{2}$ auf $\widetilde{x_{1}} \widetilde{x}_{0}$ liegen. Das ist unmöglich, da $y_{2}$ auf $\widetilde{x}_{p} \widetilde{x}_{p-1}$ liegt und $\widetilde{x}_{p}$ nicht mit $y_{2}$ übereinstimmt. Daher kann $\widetilde{x}_{1}$ nicht auf $\stackrel{\curvearrowleft}{y_{1} y_{2}}$ liegen.

Wenn $y_{1}$ auf $\widetilde{x}_{q} \widetilde{x}_{q-1}$ liegt und $\widetilde{x}_{q-1}$ nicht mit $y_{1}$ übereinstimmt, so muss 
$q-1 \leqq 0$ sein. Dann muss $\widetilde{x}_{q-1}$ auf $\hat{y}_{1} y_{2}$ liegen und daher muss $y_{2}$ auf demselben Grunde wie im Falle a) auf $\widetilde{x}_{q-1} \widetilde{x}_{q-2}$ liegen. Daraus folgt, dass $p=q-1$ oder $p=q$ sein muss.

Wenn $p=q$ ist, so muss $y_{1}$ auf $\widetilde{x}_{p} \widetilde{x}_{p-1}$ liegen. Nach Voraussetzung, dass $\widetilde{x}_{p}$ auf $\mathscr{y}_{1} y_{2}$ liegt, muss $y_{1}$ mit $\widetilde{x}_{p}$ übereinstimmen. Wenn $p=q-1$ ist, so liegt $y_{1}$ offenbar auf $\widetilde{x}_{p+1} \widetilde{x}_{p}$. Damit ist die Behauptung bewiesen.

Es seien $y_{1}$ und $y_{2}$ zwei Punkte von $k$ von der Art, dass $E_{y_{1}}$ und $E_{y_{2}}$ gemeinsame Punkte enthalten und $y_{1}$ in oben für $y_{2}$ liegt. Wenn $y_{1}$ auf $\widetilde{x}_{p+1} \widetilde{x}_{p}$ liegt und $\widetilde{x}_{p}$ auf $\mathscr{y}_{1} y_{2}$ liegt, und wenn $y_{1}$ nicht mit $\widetilde{x}_{p}$ übereinstimmt, so muss $y_{2}$ auf $\widetilde{x}_{p} \widetilde{x}_{p-1}$ liegen. Diese Behauptung kann in ganz ähnlicher Weise wie oben bewiesen werden.

3. Schritt. $x,\left\{\widetilde{x}_{n}\right\}(n=0, \pm 1, \pm 2, \ldots), \widetilde{x}$ und $\widetilde{\tilde{\partial}}$ sollen dieselben Sinne wie im 2. Schritte haben. Es sei $y$ ein Punkt auf $\widetilde{x}_{p} \widetilde{x}_{p-1}$, und $z$ sei die obere Grenze der Menge aller Punkte $y^{\prime}$ von $k$ von der Art, dass $E_{y^{\prime}}$ und $E_{y}$ gemeinsame Punkte enthalten und $y^{\prime}$ in oben für $y$ liegt, und $w$ sei die obere Grenze der Menge aller Punkte $y^{\prime \prime}$ von $k$ von der Art, dass $E_{y^{\prime \prime}}$ und $E_{z}$ gemeinsame Punkte enthalten und $y^{\prime \prime}$ in oben für $z$ liegt.

Wir beweisen nun, dass, wenn der Punkt $z$ auf $\widetilde{x}_{p+1} \widetilde{x}_{p}$ liegt, w nicht auf $\widetilde{x}_{p+1} \widetilde{x}_{p-1}$ liegen muss oder mit $\widetilde{x}_{p+1}$ übereinstimmen muss. Angenommen in der Tat, dass $w$ auf $\widetilde{x}_{p+1} \widetilde{x}_{p-1}$ liegt und nicht mit $\widetilde{x}_{p+1}$ übereinstimmt. Dann muss $w$ auf $\widetilde{\widetilde{x}_{p+1}} z$ liegen. Denn, wenn $w$ auf $\underset{z \widetilde{x}_{p-1}}{\mathscr{\sigma}}$ liegt, so muss die Begrenzung $r$ von $\mathfrak{S}_{1}$ die Vereinigunysmenge $m_{\tilde{x}_{p+1}}+m_{\tilde{x}_{p}}+m_{\tilde{x}_{p-1}}$ sein; damit muss $r$ die Vereinigungsmenge von endlicher Anzahl von Häufungskontinuen in $r$ und unzerlegbaren Kontinuen sein. Dies ist aber unmöglich im Falle A.

Nun, wenn $w$ auf $\widetilde{x}_{p+1} z$ liegt und nicht mit $\widetilde{x}_{p+1}$ übereinstimmt, so muss jeder von den in $E_{z}$ enthaltenen Punkte auch in $E_{\tilde{x}_{p}}$ und nicht in $E_{\tilde{x}_{p+1}}$ enthalten sein. Daraus folgt, dass die in $E_{\tilde{x}_{p}}$ und nicht in $E_{z}$ enthaltenen Funkte existieren. Denn, wenn $m_{\tilde{x}_{p}}$ und $m_{z}$ übereinstimmt, so muss $w$ mit $\widetilde{x}_{p+1}$ übereinstimmen. Jeder in $E_{\tilde{x}_{p}}$ und nicht in $E_{z}$ enthaltene Punkt muss in $E_{y}$ enthalten sein. Ein sowohl in $E_{\tilde{x}_{p}}$ als auch in $E_{\tilde{x}_{p+1}}$ gleichzeitig enthaltener Punkt muss daher in $E_{y}$ enthalten sein, damit müssen $E_{y}$ und $E_{\tilde{x}_{p+1}}$ gemeinsame Punkte enthalten. Das ist aber ein Widerspruch mit der Annahme, dass $z$ auf $\widetilde{x}_{p+1} \widetilde{x}_{p}$ liegt und $z$ nicht mit $\widetilde{x}_{p+1}$ übereinstimmt. Damit ist die Behauptung bewiesen.

Es sei $y$ ein Punkt anf $\widetilde{x}_{p} \widetilde{x}_{p-1}$, und $z$ sei die untere Grenze der Menge aller Punkte $y^{\prime}$ von $k$ von der Art, dass $E_{y^{\prime}}$ und $E_{y^{\prime}}$ gemeinsame Punkte enthalten und $y$ 
in oben für $y^{\prime}$ liegt, und $w$ sei die untere Grenze der der Menge aller Punkte $y^{\prime \prime}$ von $k$ derart, dass $E_{y^{\prime \prime}}$ und $E_{z}$ gemeinsame Punkte enthalten und $z$ in oben für $y^{\prime \prime}$ liegt. Wenn der Punkt $z$ dabei auf $\widetilde{x_{p-1}} \widetilde{x}_{p-2}$ liegt, so muss $w$ nicht auf $\widetilde{x}_{p} \widetilde{x}_{p-2}$ liegen oder $w$ und $\widetilde{x}_{p-2}$ übereinstimmen. Diese Behauptung kann in gleicher Weise wie oben bewiesen werden.

4. Schritt. Für beide beliebige Punkte $x$ und $x^{\prime}$ von $k$ in der in 1. Schritte

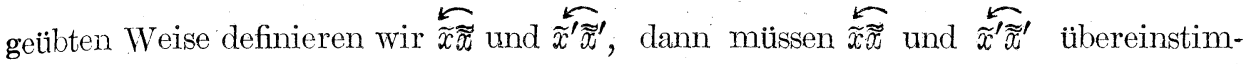
men oder höchstens nur Endpunkte gemein haben. Hierbei unterscheiden wir die beiden Fälle.

1. Fall. $\widetilde{x} \widetilde{\widetilde{x}}$ enthält mindestens einen von $\widetilde{x}^{\prime}$ und $\widetilde{\widetilde{x}}^{\prime}$ verschiedenen Punkt von $\stackrel{\mathscr{x}}{\widetilde{x_{j}}}$.

In diesem Falle in der Menge $\widetilde{x} \widetilde{\widetilde{x}}-(\widetilde{x}+\widetilde{\widetilde{x}})$ wird ein von $\widetilde{x}^{\prime}$ und $\widetilde{\widetilde{x}}^{\prime}$ verschiedener Punkt $y$ von $\widetilde{x}^{\prime} \widetilde{\widetilde{x}}^{\prime}$ enthalten. Wir können annehmen, dass $y$ in $\widetilde{x}_{p+1} \widetilde{x}_{p}$ enthalten ist, und dass $y$ nicht mit $\tilde{x}_{p}$ übereinstimmt.

Ein Punkt $A$ von $r$ sei in $E_{y}$ enthalten, und wir bezeichnen mit $y_{1}$ die obere Grenze der Menge aller Punkte $y^{\prime}$ von $k$ von der Art, dass $E_{y^{\prime}}$ den Punkt $A$ enthält und $y^{\prime}$ in oben für $y$ liegt, und wir bezeichnen auch mit $y_{2}$ die untere Grenze der Menge aller Punkte $y^{\prime \prime}$ von $k$ von der Art, dass $E_{y^{\prime \prime}}$ den Punkt $A$ enthält und $y$ in oben für $y^{\prime \prime}$ liegt. Da der Punkt $A$ in mindestens einem von beiden $E_{\tilde{x}_{p+1}}$ und $E_{\tilde{x}_{p}}$ enthalten ist, nehmen wir erstens an, dass $E_{\tilde{x}_{p}}$ den Punkt $A$ enthält. ${ }^{1)}$ Nach 2. Schritte muss dann $y_{2}$ auf $\widetilde{x}_{p} \widetilde{x}_{p-1}$ liegen. Wenn dabei $y_{2}$ nicht mit $\widetilde{x}_{p}$ übereinstimmt, so muss wiederum nach 2. Schritte der Punkt $y_{1}$ auf $\widetilde{x}_{p+1} \widetilde{x}_{p}$ liegen. Wenn $y_{2}$ mit $\widetilde{x}_{p}$ übereinstimmt, und wenn $y_{x}$ nicht auf $\widetilde{x}_{p+1} \widetilde{x}_{p}$ liegt, so muss nach 2. Schritte $z_{1}$ auf $\widetilde{x}_{p+2} \widetilde{x}_{p+1}$ liegen.

Wir bezeichnen mit $\widetilde{y}_{0}$ die unteren Grenze der Menge aller Punkte $y^{\prime}$ von $k$ von der Art, dass $E_{y_{1}}$ und $E_{y^{\prime}}$ gemeinsame Punkte enthalten und $y_{1}$ in oben für $y^{\prime}$ liegt. Hierbei unterscheiden wir zwei Fälle.

Fall a) $y_{1}^{\prime}$ liegt auf $\widetilde{x}_{p+1} \widetilde{x}_{p}$. In diesem Falle muss nach 2. Schritte $\widetilde{y}_{0}$ auf $\widetilde{x}_{p}, \widetilde{x}_{p-1}$ liegen. Denn $y_{1}$ liegt auf $\widetilde{x}_{p+1} \widetilde{x}_{p}$ und $y_{1}$ stimmt nicht mit $\widetilde{x}_{p}$ überein und $\widetilde{x}_{p}$ liegt auf $\widetilde{y_{1}} \widetilde{y}_{0}$.

1) Angenommen, dass $E_{\varepsilon_{p+1}}^{\sim}$ den Punkt $A$ enthält. In diesem Falle unterscheiden wir zwei Fälle.

1. Fall. $y$ stimmt weder mit $\tilde{x}_{p}$ noch mit $\widetilde{x_{p+1}}$ überein. In diesem Falle gilt auch die. Analogie mit der folgenden Beweisführung.

2. Fall. $y$ stimmt mit $\widetilde{x}_{p+1}$ überein. In diesem Falle gilt auch die folgende Beweisführung, wenn wir $p$ durch $p+1$ ersetzen. 
Fall b) : $y_{1}$ liegt auf $\widetilde{x}_{p+2} \widetilde{x}_{p+1}$ und stimmt nicht mit $\widetilde{x}_{p+1}$ überein. In diesem Falle muss nach 2. Schritte $\widetilde{y}_{0}$ auf $\widetilde{x}_{p+1} \widetilde{x}_{p}$ liegen. Anderseits muss $\widetilde{y}_{0}$ auf $\widetilde{x}_{p} \widetilde{x}_{p-1}$ liegen. Daher im Falle b) stimmt $\widetilde{y}_{0}$ mit $\widetilde{x}_{p}$ überein.

Wir bezeichnen mit $\widetilde{y}_{1}$ die obere Grenze der Menge aller Punkte $y^{\prime}$ von $k$ von der Art, dass $E_{\tilde{y}}$ und $E_{y^{\prime}}$ gemeinsame Punkte enthalten und $y^{\prime}$ in oben für $\widetilde{y}_{0}$ liegt. Wir unterscheiden dabei zwei Fälle.

Fall a) $\widetilde{y}_{0}$ liegt auf $\widetilde{x}_{p} \widetilde{x}_{p-1}$ und stimmt nicht mit $\widetilde{x}_{p}$ überein. In diesem Falle muss nach 2. Schritte $\widetilde{y}_{1}$ auf $\widetilde{x}_{p+1} \widetilde{x}_{p}$ liegen.

Fall b) $\widetilde{y}_{0}$ stimmt mit $\widetilde{x}_{p}$ überein. In diesem Falle muss $\widetilde{y}_{1}$ auf $\widetilde{x}_{p+2} \widetilde{x}_{p+1}$ liegen.

Schliesslich können wir in den beiden Fällen a) und b) eine Nummer $h$ hinweisen von der Art, dass $\widetilde{y}_{0}$ auf $\widetilde{x}_{h} \widetilde{x}_{h-1}$ liegt und $\widetilde{y}_{1}$ auf $\widetilde{x}_{h+1} \widetilde{x}_{h}$ liegt.

Wir definieren nun $\widetilde{y}_{n}(n=2,3, \ldots)$ wie in 1 . Schritte, so kann nach 3 . Schritte $\widetilde{y}_{2}$ nicht auf $\widetilde{x}_{h+1} \widetilde{x}_{h-1}$ liegen, wenn $\widetilde{y}_{2}$ nicht mit $\widetilde{x}_{h+1}$ übereinstimmt. Wenn $\widetilde{y}_{1}$ nicht mit $\widetilde{x}_{h+1}$ übereinstimmt, so muss nach 2 . Schritte $\widetilde{y}_{2}$ auf $\widetilde{x}_{h+2} \widetilde{x}_{h+1}$ liegen. Wenn $\widetilde{y}_{1}$ mit $\widetilde{x}_{h+1}$ übereinstimmt, so muss nach 2 . Schritte $\widetilde{y}_{2}$ auf $\widetilde{x}_{h+3} \widetilde{x}_{h+2}$ liegen. Folglich existiert eine Nummer $h_{2}$ von der Art, dass $\widetilde{y}_{1}$ auf $\widetilde{x}_{h_{2}} \widetilde{x}_{h_{2}-1}$ und $\widetilde{y}_{2}$ auf $\widetilde{x}_{h_{2}+1} \widetilde{x}_{h_{2}}$ liegt, wo $h_{2}=h+1$ oder $h_{2}=h+2$ ist. Auf demselben Grunde existiert eine Nurmmer $h_{3}$ von der Art, dass $\widetilde{y}_{2}$ auf $\widetilde{x}_{h_{3}} \widetilde{x}_{h_{3}-1}$ und $\widetilde{y}_{3}$ auf $\widetilde{x}_{h_{3}+1} \widetilde{x}_{h_{3}}$ liegt, wo $h_{3}=h_{2}+1=\left\{\begin{array}{l}h+2 \\ h+3\end{array}\right.$ oder $h_{3}=h_{2}+2=\left\{\begin{array}{l}h+3 \\ h+4\end{array}\right.$ ist. Indem wir die 2. und 3. Schritte auf $\widetilde{y}_{n}(n=4,5,6, \ldots)$ anwenden, können wir leicht folgern, dass $\widetilde{y}$ mit $\widetilde{x}$ übereinstimmt, wenn wir $\lim _{n \rightarrow \infty} \widetilde{y}_{n}$ mit $\widetilde{y}$ bezeichnen.

Die Analogie mit den oberen Betrachtungen führt nun ohne weiteres zu $\widetilde{y}_{n}(n=-1,-2, \ldots)$. Wir können daher schliessen, dass $\overline{\widehat{y}}$ mit $\widetilde{\tilde{x}}$ übereinstimmt, wenn wir $\lim \widetilde{y}_{n}$ mit $\widetilde{\widetilde{y}}$ bezeichnen. Also stimmt $\widetilde{\widetilde{y} \bar{y}}$ mit $\widetilde{\widetilde{x}} \widetilde{\widetilde{x}}$ überein. Ebenfalls

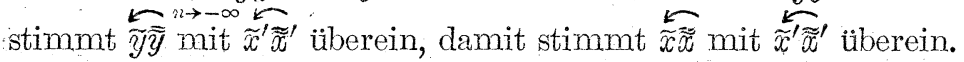

2. Fall. $\widetilde{\widetilde{x}} \tilde{\tilde{x}}$ und $\stackrel{\leftarrow}{x^{\prime}}$ gemein haben höchstens nur Endpunkte.

5. Schritt. Es sei $l$ ein fundamentales Intervall ${ }^{1)}$ von $k$, so entsprechen höchstens abzählbar viele Punkte $y_{1}, y_{2}, y_{3}$, ... von $l$ den einfachen Primenden von $\mathfrak{S}_{1}$ und ein zu mehrfachem Primende entsprechender Punkt $x$ ist im oben definierten $\widetilde{x} \widetilde{\tilde{x}}$ enthalten. Nach 4. Schritte existiert höchstens abzählbar viele voneinander verschiedene $\widetilde{\widetilde{x}} \widetilde{\widetilde{x}}$. Es sei $z$ beliebiger Punkt auf einem $\widetilde{x} \widetilde{\widetilde{x}}$, so ist $\sum m_{z}(z \in \widetilde{x} \widetilde{x})$ die Vereinigungsmenge von höchstens abzählbarer Anzahl von Häu-

1) K. Koseki. (II). 
fungskontinuen in $r$ und unzerlegbaren Kontinuen $m_{\tilde{x}_{n}}(n=0, \pm 1, \pm 2, \ldots)$ und $m \tilde{x}, m \tilde{\tilde{x}}$. Daher muss $r$ die Vereinigungsmenge von höchstens abzählbarer Anzahl von Häufungskontinuen in $r$ und unzerlegbaren Kontinuen, wenn $r$, , monostratique “ ist. Folglich muss im Falle A $r$ nicht ,, monostratique " sein.

Fall B. $r$ sei die Vereinigungsmenge von höchstens abzählbarer Anzahl von Häufungskontinuen in $r^{*}$ und unzerlegbaren Kontinuen.

In diesem Falle ist $r$, monostratique“. Angenommen in der Tat, dass $r$ nicht „monostratique" ist, dann lässt sich $r$ in "Tranches fondamentales " zyklisch zerlegen derart, dass $\gamma=\sum T_{x}$ ist. Dabei bedeutet $T_{x}$ ein , Tranche fondamentale ", und Suffix $x$ durchschreitet eine Kreisperipherie und aus der Beziehung $\lim _{n \rightarrow \infty} x_{n}=x_{0}$ folgt die Beziehung limsup $T_{x_{n}} \subset T_{x_{0}}$. Wenn $t$ ein Teilkontinuum von ${ }^{r \rightarrow \infty}$ ist, und wenn $t$ die beide Punkte $A \in T_{x_{1}}$ und $B \in T_{x_{2}}$ enthält, wo $x_{1} \neq x_{2}$ ist, so muss $t$ mindestens eines von den beiden $\sum_{x_{1}<c<x_{2}} T_{x}$ und $\sum_{x_{1}<x<x_{2}} T_{x}$ enthalten. ${ }^{1>}$ Daraus folgt ohne weiteres, dass, wenn $t$ ein Häufungskontinuum in $r$ oder ein unzerlegbares Kontinuum ist, $t$ in einem und demselben , Tranche fondamentale " enthalten sein muss. Daher kann $r$ nicht die Vereinigungsmenge von höchstens abzählbarer Anzahl von Häufungskontinuen in $r$ und unzerlegbaren Kontinuen sein, wenn $r$ nicht , monostratique“ ist. Damit ist die Behauptung bewiesen.

Zusatz. Es sei $r$ die beschränkte und gemeinsame Begrenzung zweier Gebiete $\mathfrak{S}_{1}$ und $\mathfrak{G}_{2}$ und $T_{x}$ sei ein , Tranche fondamental ". Dann besteht $T_{x}$ aus höchstens abzählbarer Anzahl von Häufungskontinuen in $r$ und unzerlegbaren Kontinuen.

Satz III. Es sei $r$ die beschränkte und gemeinsame Begrenzung zweier Gebiete $\mathfrak{S}_{1}$ und $\mathfrak{S}_{2}$. Wenn $r^{*}$ nicht „, monostratique " ist, so ist jedes , Tranche fondamentale " von $r$, ausser höchstens abzählbar vielen ,, Tranches fondamentales ", ein Häufungskontinuum in $r$.

Beweis. Satz XII in meiner letzten Arbeit (II) zeigt, dass jedes , Tranche fondamentale" von 1 , ausser höchstens abzählbar vielen "Tranches fondamentales", höchstens einen von $\mathfrak{G}_{1}$ aus erreichbaren Punkt. Daraus folgt ohne weiteres der Satz III.

Zum Schlusse möchte ich Herrn Professor T. Matumoto für seine Kritik und verschiedene wichtige Ratschlage herzlichst danken.

1) C. Kuratowski: Sur la structure des frontières communes à deux régions. Fund. Math. 12 (1928). 


\section{Literaturverzeichnis.}

C. Carathéodory. Über die Begrenzung einfach zusammenhängender Gebiete. Math. Ann. 73 (1913).

K. Koseki. Über die Begrenzung eines besonderen Gebietes, (II). Jap. Journ. Math. 19 (1948). O. Kuratowski. Sur la structure des frontières communes à deux régions, Fund. Math. 12 (1928).

A. Rosenthal. Teilung der Ebene durch irreduzible Kontinua. Sgb: Bay. Ak. Wiss. (1919). 